\title{
Visualization of Multivariate Data on Surfaces
}

\author{
Allan Rocha \\ Usman Alim \\ Mario Costa Sousa \\ Department of Computer Science \\ University of Calgary \\ Calgary, Canada \\ Email: \{acarocha, ualim, smcosta\}@ucalgary.ca
}

\begin{abstract}
This research ${ }^{1}$ builds upon ideas introduced and discussed many years ago that focus on the problem of visualizing multiple attributes on surfaces in a single view. Here we present a new perspective to this problem as well as a solution that allows us to design, visualize and interact with multivariate data on surfaces. Building upon multidisciplinary aspects, we present a new way to visualize multivariate data on surfaces by exploiting the concept of layering. First, we introduce a new real-time rendering technique and the concept of Decal-Maps, which fills a gap in the literature and allow us to create 2D visual representations such as glyphs that follow the surface geometry. Building on this technique, we propose the layering framework to facilitate the multivariate visualization design on surfaces. The use of this concept and framework allows us to connect and generalize concepts established in flat space, such as 2D maps, to arbitrary surfaces. Decal-maps opens up other new possibilities such as the use of interaction techniques. Here we demonstrate this potential by introducing a new interaction technique that allows us to explore multivariate data and to create customized focus+context visualizations on surfaces. This is achieved by introducing a new category of lenses, Decal-Lenses, which extends the concept of magic-lenses from flat space to general surfaces. Finally, this thesis showcases the process of multivariate visual design and data exploration through a series of examples from several domains such as Medicine and Geology.
\end{abstract}

\section{INTRODUCTION}

In several domains, experts commonly face the problem of investigating complex tridimensional data. This tridimensional data is often multifaceted: for example multivariate, consisting of several independent properties such as pressure, temperature; multimodal, data obtained from different modalities like Computed Tomography (CT) and Magnetic Resonance Imaging (MRI) acquisition processes; or multirun, the data generated from several simulation runs, such as climate simulations used for weather forecast. Due to the complexity and heterogeneity of such datasets, visualization techniques are employed to facilitate exploration and better understanding for future decision making tasks.

Visualizing tridimensional data inevitably leads to occlusion and clutter since a large amount of information is projected to the screen [2], [3], which is one of the reasons why tridimensional data is usually restricted to surfaces of interest. These surfaces can embed one or more attributes that need to be interpreted and correlated by domain experts for decision making. For example, in the context of Geology, experts simultaneously analyse multiple geological attributes (.e.g, rock type, porosity, permeability) bounded by sub-surfaces

\footnotetext{
${ }^{1} \mathrm{Ph} . \mathrm{D}$. thesis [1] conducted between September 2013 and March 2019
}

of the Earth that characterize the geological field. However, visualizing multiple attributes in a single view is a well-known challenge [2]: one is required to determine what to visualize and how to visualize [4].

The problem of visualizing multivariate data on surfaces has been investigated since the early '90s, starting with Crawfis' paper, "A Scientific Visualization Synthesizer" [5]'. A decade later, Taylor [6] attempted a similar approach to visualize multiple fields on height maps coming from scannedprobe microscopy data. In the previous scenarios, authors highlighted that the ideas and solutions were limited 2-3 attributes even considering simple height-map surfaces. Later, other techniques such as line integral convolution (LIC) [7] were introduced and broadly used in Visualization, however, the technical limitations still persist mostly due to the fact that addressing this problem involves multidisciplinary aspects and create multivariate visualizations on surfaces using glyphs and other representations is a challenging process.

This thesis addresses the open problem of visualizing multivariate data on surfaces by bringing methodologies for visual design that have worked well on planes (in 2D) and other contexts to general surfaces. This is achieved through a set of techniques that allow us to map visual representations to arbitrary surfaces, to apply the concept of layering similarly to how it is done in $2 \mathrm{D}$, and extend the use of interaction techniques to surfaces. This thesis details several contributions to multivariate visualization, the main ones of which we list below:

- A visual design framework to create multivariate visualizations for spatial data defined on surfaces. This framework is built from the connection between several theories and ideas from the point of view of both information and scientific visualization.

- The concepts of decals and decal-maps for multivariate visualization. These concepts allow us to extend the concept of layering and map 2D visual representations to surfaces, facilitating the process of visual design and multidisciplinary collaboration in multivariate visualization.

- An abstract layering framework to create layered visualizations on surfaces. We provide a layering framework that is based on the real-time rendering pipeline and that is independent of the surface representation and topology.

- The concept of decal-lenses for multivariate visualization. This new category of lenses adapts to the surface geometry and is suitable for managing and augmenting 
multivariate data in all stages of the visualization pipeline.

- A set of examples and an in-depth application in Geoscience that illustrates and informs how visual encodings of spatial data and the process of layering can be designed in order to create expressive multivariate visualizations on surfaces.

\section{EXTENDING THE CONCEPT OF LAYERING ON SURFACES}

We focus on the superimposition paradigm to create multivariate visualizations on surfaces. Here we refer to this paradigm as the process of layering, which is inspired by the painting metaphor [8]. The process of layering in 2D has been commonly applied in Visualization to facilitate multivariate data analysis. It consists of a multiple pass rendering process where each layer representing one or more attributes rendered in a certain order (e.g., Google Maps). The visualization of layered attributes is then facilitated by exploiting the empty space between visual elements (e.g. lines, colors, glyphs or text).

\section{A. 2D Representations on Surfaces}

By reviewing and analysing the application of the layering concept, we have identified one of the main challenges related to its use for multivariate surface data: the design and mapping of visual representations such as glyphs or textures to surfaces. Another challenge is the lack of a framework that can be used to create multiple attribute layers and that can be applied to arbitrary surfaces. Therefore, following the previous analysis and referring back to the problem statement of extending the concept of layering to surfaces, a natural research question to ask is the following: How can we design and map visual representations that follow the surface curvature?

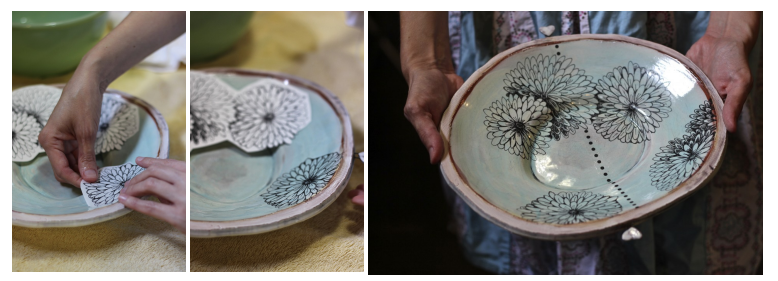

Fig. 1. Application of decals to ceramics by the artist [9] (used with permission).

When answering this question, our rationale implies that we need to design $2 \mathrm{D}$ visual representations to encode one of more data attributes and then map them locally to a surface. Fortunately, visual elements with such characteristics exist and are known as decals. In the real world, a decal is a plastic, cloth, paper or ceramic substrate that has printed on it a pattern or image that can be moved to another surface upon contact, usually with the aid of heat or water. This process is called decalcomania and was invented by the french engraver Simon François Ravenet [10]. Figure 1 illustrates the process of decalcomania. On the other hand, in the digital world, the process of "decaling" has been considered in Computer Graphics, since the work of Pedersen [11] who originally named decals as 'patchinos', when artists want to decorate 3D models' surfaces by locally applying texture patterns or other visual representations [12].

\section{DECALS AND DECAL-MAPS CONCEPT}

This thesis establishes the potential use of decals for multivariate visualization, which prior to my investigation has not been considered and formalized. Therefore, in the context of visualization we define decals as:

Definition. A decal is a 2D image where a pattern, texture, glyph or any other visual representation is defined in order to be locally mapped to a surface with the goal of representing one or more data attributes.

We have identified using decals as a powerful and natural abstraction that are suitable not only for decorating specific regions of a surface but also to model visual representations that can encode data attributes. This process of design and data encoding is highly facilitated by the fact that decals are $2 \mathrm{D}$ representations. Once the design is defined, the decal can then be transferred to the surface through a suitable mapping function. The mapping between attributes and visual representations (such as a transfer function) is what we define as decal-maps. Decal-maps can be composed by a set of decals and its multiple visual representations.

In order to place decals on surfaces, we need a texture mapping technique with the following three requirements. 1) It should be independent of the surface parametrization (texture coordinates) since many scientific datasets are not represented parametrically; 2) it should not rely on topological information from the underlying mesh such as geodesic distance because this is expensive to compute and depends on the mesh type; and 3) it should be efficient as we need a high number of decals to represent data attributes in several locations of the surface. In the next section, we define formally how decals are mapped to surfaces.

\section{A. Sphere Masking and Local Parametrization}

We begin with a surface on which we want to place decals. Let us denote this surface as $M$. The first problem we need to tackle is the one of building a parametrization of this surface that would allow the placement of decals, one at a time in an efficient manner. Since decals are small, such a parametrization need not be global. Therefore, for each decal, we build a local parametrization on $M$ that is defined only inside a Euclidean ball containing the decal.

Let us assume that the intersection of the surface $M$ and a ball $B_{c}$, centered at the point $c$ in $M$, is a disc $D_{c}:=B_{c} \cap M$. The disc $D_{c}$ may be understood as a patch of $M$ over which a decal is to be placed. We refer the reader to Fig. 2 to illustrate the discussion that follows.

Let us consider the tangent plane of surface $M$ at point $c$, which we denote as $T_{c} M$. On such a tangent plane, we can define a Cartesian coordinate system given by a previously chosen orthogonal basis $\left\{\hat{u}_{c}, \hat{v}_{c}\right\}$, for which any point $x \hat{u}_{c}+$ $y \hat{v}_{c}$ in $T_{c} M$ can be mapped to a pixel $\{x, y\}$ in the texture. 


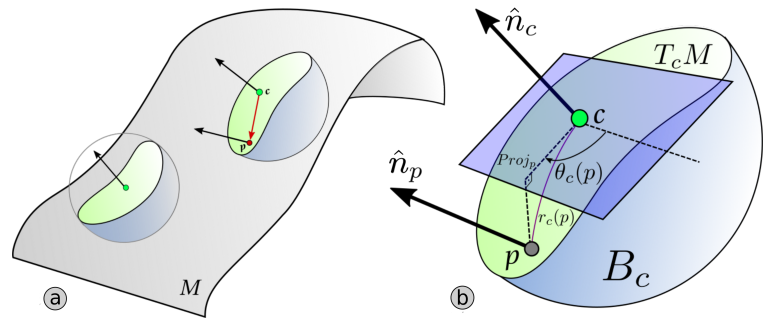

Fig. 2. Sphere masking approach: (a) Patch $D_{c}=B_{c} \cap M$, shown in green. (b) Polar coordinate system on patch $D_{c}$. Angular coordinate $\theta_{c}(p)$ : angle between the projection of $p$ on the tangent plane $T_{c} M$ and a reference vector. Radial coordinate $r_{c}(p)$ : approximates the geodesic distance from $c$ to $p$.

The orthogonal basis $\left\{\hat{u}_{c}, \hat{v}_{c}\right\}$ can be either a fixed a priori choice (e.g. randomly chosen) or computed from the data.

The problem of texture mapping then becomes the problem of matching points $p$ in the patch $D_{c} \subset M$ to pixels in the decal. Since we are assuming that the patch $D_{c}=$ $B_{c} \cap M$ is a disc, we can choose a radial coordinate system in $D_{c}$. Any point $p$ in the patch $D_{c}$ has the coordinates $p=\left(r_{c}(p), \theta_{c}(p)\right)$. Point $p$ is then mapped to the pixel $(x(p), y(p))$ in $T_{c} M$ for which $r_{c}(p)=\sqrt{\left(x(p)^{2}+y(p)^{2}\right)}$, and $\theta_{c}(p)=\arctan (x(p), y(p))$. Since $D_{c}$ is a disc, these equations always have a solution $\{x(p), y(p)\}$.

The angle $\theta_{c}(p)$ can be obtained by simply projecting $p$ onto $T_{c} M$ and computing the angle of this projection with a preselected vector in $T_{c} M$, e.g. $\hat{u}_{c}$. The radius $r_{c}(p)$, however, is given by the geodesic distance between $c$ and $p$ in $M$, which may be expensive to compute in the general case. Because decals are supposed to be small compared to the surface $M$, we can use approximations of the geodesic distance to estimate $r_{c}(p)$. In this thesis we analysed three distances: euclidean distance, cosine interpolation and the Isophotic distance. We refer the reader to the Decal-Maps paper [13] for more details.

\section{LAYERING FRAMEWORK}

Based on the previous definitions, we were able to design the layering framework to map and visualize decals on surfaces. Our algorithm is independent of the surface representation and allows a high number of decals to be rendered at interactive frame rates. The visual mapping for each attribute defines which type of representation will be used and organized in layers. For example, one attribute can be mapped to a colormap and another one to a decal-map which leads to two layers of information.

To render decals on surfaces we introduce a new real-time rendering technique. We explore the graphics pipeline using OpenGL and GLSL. Our implementation to create a surface layer consists of a multi-pass approach which is divided into three main steps: 1) compute the geometry buffer (G-buffer); 2) compute the sphere masking buffer (SM-buffer); and 3) apply the decals on the surface (decal mapping) [13]. In summary, Fig. 3 illustrates each step of our technique applied to a bumpy sphere.

\section{A. Multivariate Geological Data Visualization}

Now we demonstrate the use of the Decal-Maps technique to multivariate visualization design. We present an application

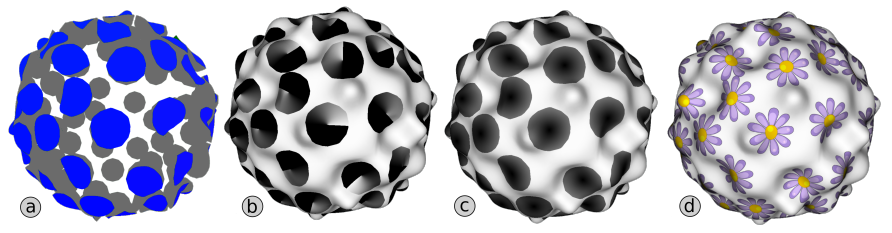

Fig. 3. Steps of our implementation: (a) Sphere masking; (b) Angular coordinate; (c) Radial coordinate; (d) Decal mapping.

of the layering process to a geological reservoir model. We introduced for the first time in this domain, a multivariate illustrative visualization of a reservoir simulation model combining six attributes: rock type, porosity, oil flow direction and magnitude, and water flow direction and magnitude. We visualize these attributes on the surface of the hexahedral grid. Even though reservoir grids may have degenerate cells (to embed geological features such as faults), our technique works well since it does not depend on the surface representation.

The data consists of a black oil simulation conducted by a domain expert. The main goal of this simulation is to analyze the process of oil recovery. During the simulation, water is injected at a location of the reservoir (using an injection well) to push the oil to another location from where it is to be extracted (using a production well). Oil migration depends on other properties such as porosity, rock type and permeability, reinforcing the need to provide an integrated multivariate visualization.

Table I shows a summary of our visualization design. Many of the design ideas come from initial discussions with domain experts, literature review as well as inspiration from geological illustrations. Figure 4 illustrates the layering process that we describe as follows.

$$
\begin{array}{rl|}
\text { geological data } & \text { visual mapping } \\
\text { rock type } & \text { pastel colormap } \\
\text { porosity } & \text { proximity between decals } \\
\text { oil flow direction } & \text { red arrow decal } \\
\text { oil flow magnitude } & \text { decal transparency and size } \\
\text { water flow direction } & \text { blue arrow decal } \\
\text { water flow magnitude } & \text { decal transparency and size } \\
& \text { TABLE I }
\end{array}
$$

MAPPING GEOLOGICAL DATA ATTRIBUTES TO VISUAL REPRESENTATIONS.

Rock type layer: Within reservoir models, rock type is represented as a set of indices. Thus, the visual variable suitable for rock type is the one used to represent categorical data (no intrinsic ordering) [14]. Since each reservoir usually has just a few different rock types, we use a pastel colormap to represent each rock type.

Porosity layer: Porosity is a volumetric quantity expressed as a percentage that measures the capacity of rocks to store fluids [15]. Naturally, visual variables such as value and position can be used for indicating high and low values of porosity. In our case, we adopt position; highly clustered areas indicate low porosity and vice versa. Our inspiration for this choice comes from traditional illustrations where porosity is represented as a set of rock grains packed in a certain configuration.

Oil flow layer: Oil flow is visualized using a red arrow 

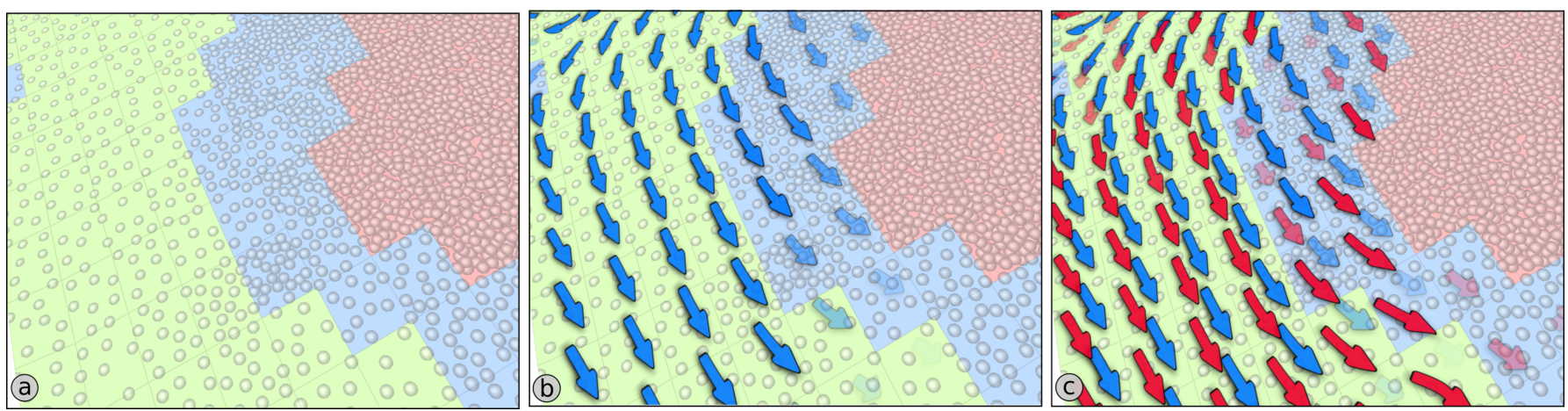

Fig. 4. Layering on the surface of a reservoir model combininig rock type, porosity, and oil and water flow.

decal-map. This creates a high contrast with the rock type and porosity background. Red is also a conventional choice for visualizing oil (domain expert feedback). In our design, we orient the arrow decal to follow the oil flow direction [13], whereas the magnitude is used to control the size and transparency of the decal. We use both size and transparency due to the fact that the size of the decals is altered due to perspective projection.

Water flow layer: For water flow, we use a design similar to the oil flow layer. We use a blue arrow (domain expert feedback) decal-map to visualize water flow direction as well as size and transparency to encode water flow magnitude.

Decal placement: We need to consider decal placement strategies for porosity, oil and water flow. For the porosity distribution, we map the porosity values (generally between $0-0.3(30 \%)$ ) to proximity using an importance driven Poisson sampling technique [16]. We slightly overlap the 'grains' to increase the sense of connectivity [14]. For the placement of the oil and water flow decals, we use the quad faces of the reservoir grid. We place a decal at the center of each triangle of the quad face. This helps decrease decal overlapping between the layers. To increase the sense of flow and connectivity, we slightly overlap the arrow decals with each other.

Last but not least, our visualization represents the data in an illustrative fashion. It is intuitive to visualize porosity using grains, flow using arrows and rock type via color. A quick glance of the visualization can tell a lot about the phenomenon thus facilitating the interaction between professionals from different backgrounds. A suplementary video material illustrating the process the layering can be found here ${ }^{2}$.

\section{Focus+CONTEXT AND Interaction TeChNiques FOR VISUAL MANAGEMENT}

We define visual management as the act of manipulating the visualization at any stage of the visualization pipeline in order to reduce clutter, occlusion or visual interference to facilitate data understanding. Even in 2D, the process of layering may lead to clutter and visual interference due to the sheer amount of data and the limitations of our visual system since visualizing all available data is not a solution as it can mislead and confuse users during data interpretation. This is

\footnotetext{
${ }^{2}$ https://vimeo.com/188876381
}

why concepts such as focus+context, details-on-demand, and clustering techniques are employed in Visualization [17]. Such techniques create several layers of abstraction by augmenting and managing visual information for a better data comprehension and exploration [4]. Moreover, this process of visual management allows multivariate visualizations to scale, since visual information can be ignored, reduced, or summarized in order to support a domain task or interaction.

Similar to the assumption that the process of layering and visual design can be extended from 2D visualizations to surfaces, we envision that interaction techniques and other paradigms well established in 2D visualization can be extended to surfaces, to enhance and manage multivariate data. This research provides an example of such possibility by introducing a new focus+context technique based on the concept of magic lenses [18]. In visualization, lenses are frequently employed for on-demand data exploration [19]. Therefore, we introduce Decal-Lenses -interactive lenses that follow the surface geometry - with the goal of customizing superimposed multivariate visualizations, i.e., by managing or augmenting visual representations of attributes displayed at different locations of the surface. The concept of a decal-lens fills a gap in the literature by introducing a new lens paradigm that is suitable to operate over surfaces or even 3D datasets, if the later is augmented with support surfaces to hold the lenses [20].

\section{DECAL-LENSES CONCEPT}

Our lenses are patches of 2D manifolds built to attach smoothly to non-flat surfaces. To the best of our knowledge, such lenses have not been previously defined, and they do not fit in the previously discussed classification [19] of 2D lenses, 2.5D lenses, and 3D lenses. Metaphorically, our lenses resemble 2D decals drawn over a surface; we, therefore, denote them as decal-lenses. However, decals and decal-lenses are fundamentally different; decals are textures stamped onto surfaces (e.g., to encode data attributes), whereas decal-lenses are surface regions (patches) designed to allow for a wider range of uses (focus+context) and interactions. Some examples are userdefined placement (decal-lenses must be amenable to drag and drop operations at the user's will), multi-lens composition (when different lenses are superimposed, their output must be 


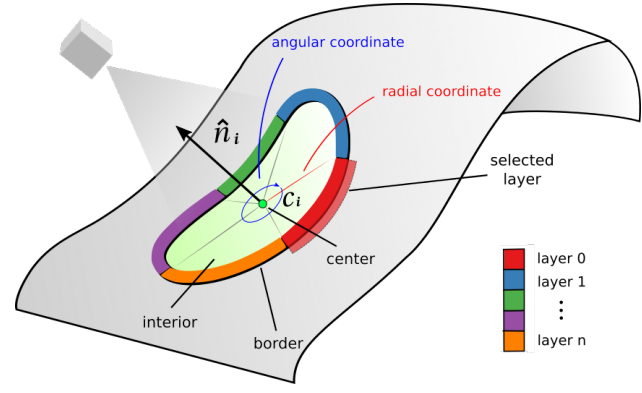

Fig. 5. Conceptual illustration of our approach. The Lens interior filters attributes represented as layers. The border displays each attribute represented as a color. To design the border, we take advantage of the local polar coordinate parametrization of the lens. A local camera can also be associated with the lens.

either filtered or combined), and lens interaction (the user may interact with the lenses to change their properties).

\section{A. Definition}

We refer the reader to Fig. 5 to illustrate the concepts we now describe. We denote the surface on which we will place the lenses as $M$. The user first picks a point $c$ on the surface, which is used as the center of the lens about to be placed. Given the selected point, a normal vector $\hat{n}$ is computed (by averaging neighboring normal vectors); this normal vector is later used to position a local camera upon a user's request. We denote as $B_{c}$, a ball centered at $c$. The intersection of the surface $M$ and $B_{c}$ defines a patch $P_{c}:=B_{c} \cap M$. In the context of decals, in Sec III we denote this intersection as sphere masking and assume that this patch is a disk since decals are small and require a local parametrization for texture mapping. In the case of a decal-lens, the patch $P_{c}$ may be understood as the region of $M$ that will contain the lens and may not be a disk. The size of the lens is defined by the user-defined radius of the ball $B_{c}$.

To parametrize $P_{c}$, we can choose a radial coordinate system. Any point $p$ in the patch $P_{c}$ has the coordinates $p=\left(r_{c}(p), \theta_{c}(p)\right)$. The angular coordinate $\theta_{c}(p)$ is simply computed by using a reference vector in the tangent space of the surface, which is an arbitrary choice. The radial coordinate $r_{c}(p)$ is given by an approximation of the geodesic distance of the surface patch. Here, we use the cosine approximation [13] for sphere-like surfaces and the Euclidean distance of the 3D space for more complex geometries.

The border of the lens is designed as the ring obtained from a pre-defined range of the radial parametrization. By dividing the angular coordinate, the lens' border can be divided into as many pieces as there are properties to select. These pieces are ordered according to the reference vector in the tangent space. The active property has its respective part of the lens' border made wider by properly scaling the radial coordinate of the local parametrization. The radial coordinate is also used to blend the contents of superimposed lenses.

\section{B. Geo-Visualization Using Decal-Lenses}

To illustrate the use of Decal-Lenses, we use a multivariate Earth dataset as an example. Such data is traditionally visu-

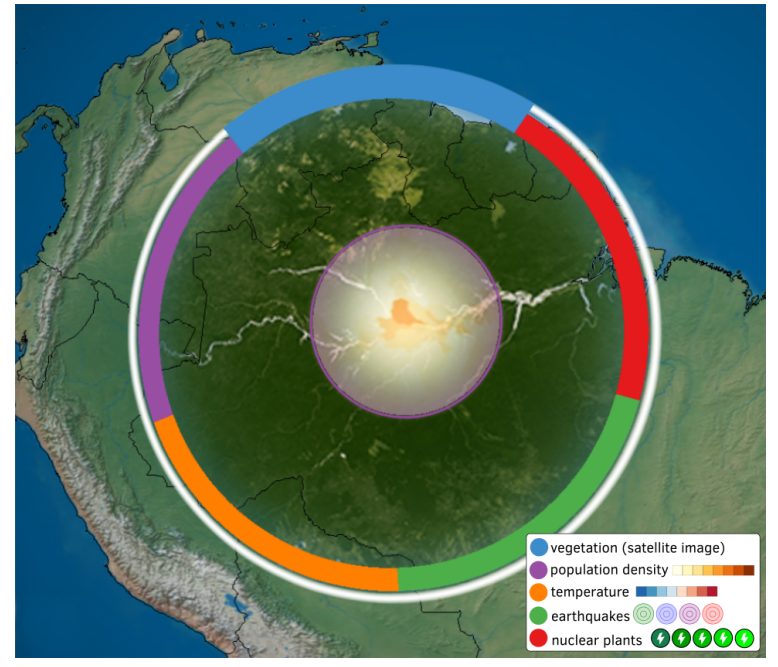

Fig. 6. Multivariate geo-visualization. A decal-lens is used as context to display vegetation density and another as focus illustrating population density in the capital state area (Manaus) along the Amazon river; the color wheel represents five attributes.

alized and interpreted as layers of 2D maps using geographic information systems (GIS) (e.g., atmospheric data, satellite images, and socioeconomic data). Here, we visualize such layers on a Digital Earth model to avoid distortions created by projecting the Earth surface into the plane, which can lead to erroneous interpretation of length and area measurements [21]. However, the spherical nature of the Earth has inherent challenges related to modeling and visualization. Here, we demonstrate the use of decal-lenses for multivariate data exploration on the Earth surface.

For our visualization, we consider the following attributes: (raster data) vegetation density, temperature and population density; and (point data) nuclear plants and earthquakes. Vegetation density is represented from a satellite image; whereas temperature and population density are represented using colormaps; cool-to-warm diverging and light-to-dark orange respectively. Using decal-maps, we represent nuclear plants and earthquakes similarly to Rocha et al. [13]. Figure 6 illustrates the use of decal-lenses to manage information. Following our definition, a decal-lens is placed on the surface and the wheel controls the layers of information displayed in the lens interior. The user can drag and resize the lens as well as compose multiple lenses for better data analysis.

\section{EXTENSIONS \& RELATED CONCEPTS}

The simplicity of our approach allows us to extend decallenses and use them in combination with other concepts. In the Decal-Lenses paper [20] we focus on three main approaches: (1) local cameras, each lens has an attached camera which is automatically positioned by using the average normal direction around the center (Figure 5); (2) operations over multiple decal-lenses, we can easily abstract the decal-lenses concept to create arbitrary shaped lenses using brushing and lassoing sketching operations over surfaces; and (3) the concept of support surfaces, which are surfaces created with the goal of augmenting the use of interaction techniques. The description 
of these techniques is quite extensive and we refer the reader to the Decal-Lenses paper [20] for more details. Moreover, the supplementary video material can be found here ${ }^{3}$.

\section{DEMONSTRATION EXAMPLES AND APPLICATIONS}

This research illustrates the use of the proposed techniques in a variety of demonstrations examples. Our goal is to inform visualization practitioners how to design and apply the proposed multivariate visualization concepts. Moreover, our techniques were also applied and extended in two application scenarios. In [22] we proposed a multivariate visualization over isosurfaces extracted from oceanography data. In [23], we proposed a novel illustrative multivariate visualization for geological modelling. Supplementary video material for both applications can be accessed here ${ }^{4}$.

\section{CONCLUSION}

There has been a growing effort on bringing concepts from information visualization to scientific visualization. We have also added to these efforts by emphasizing the importance of the design process in multivariate visualization, extending the concept of layering on surfaces [13], [20], [22], [23], and introducing to Scientific Visualization an interaction technique inspired by the magic lenses concept that is well established in information visualization [20].

However, many more techniques that implement effective visual abstractions, concepts for overview-detail, data comparison and correlation, spatial to abstract data integration, and interaction techniques for data manipulation are still either incipient or not available in the scientific visualization community. We argue that exploring these ideas born in the information visualization community and other areas of expertise in the eyes of scientific visualization can provide a fruitful avenue of research and creative solutions in the years to come. We hope that this thesis can provide a solid example that can be used as inspiration for those who aim to pursue this path in the future.

\section{Publications and Awards}

During this Ph.D. work, we achieved three journal publications and one conference paper. Two papers were published at the journal IEEE Transactions of Visualization and Computer Graphics [13], [20] (Qualis A1), one at the journal Computer Graphics Forum (Qualis A1) [23]. A conference paper [22] was published at the Visualization in Environmental Sciences workshop (EnvirVis) co-located with EuroVis 2017. This conference paper also won the Canadian visualization contest Visualize This! ${ }^{5}$, promoted by Compute Canada. As first prize, we were also invited to present this work at the HPCS $2017^{6}$.

\section{ACKNOWLEDGMENT}

This research was supported in part by the NSERC/AITF/FCMG Industrial Research Chair Program in

\footnotetext{
${ }^{3}$ https://vimeo.com/295735599

${ }^{4}$ https://vimeo.com/224362432 and https://vimeo.com/278025479

${ }^{5}$ https://www.computecanada.ca/events/visualization-challenge/

${ }^{6}$ High Performance Computing Symposium - http://2017.hpcs.ca/
}

Scalable Reservoir Visualization and by Discovery Grants from NSERC.

\section{REFERENCES}

[1] A. Rocha, "Visualization of multivariate data on surfaces," Ph.D. dissertation, University of Calgary, 2019.

[2] R. Fuchs and H. Hauser, "Visualization of multi-variate scientific data," in Computer Graphics Forum, vol. 28. Wiley Online Library, 2009, pp. $1670-1690$.

[3] J. Kehrer and H. Hauser, "Visualization and visual analysis of multifaceted scientific data: A survey," IEEE Transactions on Visualization and Computer Graphics, vol. 19, no. 3, pp. 495-513, 2013.

[4] I. Viola and T. Isenberg, "Pondering the concept of abstraction in (illustrative) visualization," IEEE Transactions on Visualization and Computer Graphics, vol. 24, no. 9, pp. 2573-2588, Sept 2018.

[5] R. A. Crawfis and M. J. Allison, "A scientific visualization synthesizer," in Proc. of Vis. IEEE, 1991, pp. 262-267.

[6] R. Taylor, "Visualizing multiple fields on the same surface," IEEE Computer Graphics and Applications, vol. 22, no. 3, pp. 6-10, 2002.

[7] J. J. van Wijk, "Spot noise texture synthesis for data visualization," in Proceedings of the 18th Annual Conference on Computer Graphics and Interactive Techniques, ser. SIGGRAPH '91. New York, NY, USA: ACM, 1991, pp. 309-318. [Online]. Available: http://doi.acm.org/10.1145/122718.122751

[8] R. M. Kirby, H. Marmanis, and D. H. Laidlaw, "Visualizing multivalued data from $2 \mathrm{~d}$ incompressible flows using concepts from painting," in Proc. of Vis. IEEE, 1999, pp. 333-340. [Online]. Available: http://dl.acm.org/citation.cfm?id=319351.319429

[9] Julie Guyot, http://www.julieguyotstudio.com/, 2019, Online; accessed 18-Feb-2019.

[10] P. Wandless, Image Transfer on Clay: Screen, Relief, Decal \& Monoprint Techniques, ser. A Lark Ceramics Book Series. Lark Books, 2006. [Online]. Available: https://books.google.ca/books?id= yihUAAAAMAAJ

[11] H. K. Pedersen, "Decorating implicit surfaces," in Proceedings of the 22nd annual conference on Computer graphics and interactive techniques. ACM, 1995, pp. 291-300.

[12] R. Schmidt, C. Grimm, and B. Wyvill, "Interactive decal compositing with discrete exponential maps," ACM Transactions on Graphics (TOG), vol. 25 , no. 3, pp. 605-613, 2006.

[13] A. Rocha, U. Alim, J. D. Silva, and M. C. Sousa, "Decal-maps: Realtime layering of decals on surfaces for multivariate visualization," IEEE Transactions of Visualization and Computer Graphics, vol. 1, 2017.

[14] C. Ware, Information visualization: perception for design. Elsevier, 2013.

[15] Lyons and Plisga, Standard Handbook of Petroleum and Natural Gas Engineering. Elsevier Science, 2011. [Online]. Available: https://books.google.ca/books?id=h-DRjBCI08QC

[16] M. Corsini, P. Cignoni, and R. Scopigno, "Efficient and flexible sampling with blue noise properties of triangular meshes," IEEE TVCG, vol. 18, no. 6, pp. 914-924, 2012

[17] B. Shneiderman, "The eyes have it: A task by data type taxonomy for information visualizations," in Visual Languages, 1996. Proceedings., IEEE Symposium on. IEEE, 1996, pp. 336-343.

[18] E. A. Bier, M. C. Stone, K. Pier, W. Buxton, and T. D. DeRose, "Toolglass and magic lenses: the see-through interface," in SIGGRAPH '93. ACM, 1993, pp. 73-80.

[19] C. Tominski, S. Gladisch, U. Kister, R. Dachselt, and H. Schumann, "Interactive lenses for visualization: An extended survey," in Computer Graphics Forum, 2016.

[20] A. Rocha, J. D. Silva, U. R. Alim, S. Carpendale, and M. C. Sousa, "Decal-lenses: Interactive lenses on surfaces for multivariate visualization," IEEE Transactions on Visualization and Computer Graphics, vol. to appear, pp. 1-1, 2018.

[21] A. Mahdavi-Amiri, T. Alderson, and F. Samavati, "A survey of digital earth," $C \& G$, vol. 53, pp. 95-117, 2015.

[22] A. Rocha, J. D. Silva, U. Alim, and M. C. Sousa, "Multivariate Visualization of Oceanography Data Using Decals," in EnvirVis. The Eurographics Association, 2017, pp. 31-35.

[23] A. Rocha, R. C. R. Mota, H. Hamdi, U. R. Alim, and M. C. Sousa, "Illustrative multivariate visualization for geological modelling," $C G F$, vol. 37, no. 3, 2018. 